\title{
Design of Environment Monitoring System Based on GPRS and ARM
} Zhao Cui ${ }^{1, a}$, Gong Zhi ${ }^{2, b}$

${ }^{1}$ Department of Information Engineering, Wuhan Business University, Wuhan, 430056, China

${ }^{2}$ Department of research and development, China Shipbuilding Industry Corp No. 722 Research Institute, Wuhan, 430079, China

aemail: upzhao@yeah.net bemail: forzazhi@163.com

Keywords: Environment Monitoring; GPRS; ARM; Real-time

\begin{abstract}
For the problems of our country's environment monitoring system, an environment monitoring system based on GPRS and ARM techniques is designed in this paper. The monitor uses LPC2220 as its control core which is an ARM7 microcontroller, sensors collect data of SO2, temperature and humidity, and then the GPRS module transfers these data according the GPRS to the monitoring center software which is on the server.
\end{abstract}

\section{Introduction}

In recent years, with the development of China's economic and more and more serious damage of natural ecological environment, the requirement of environmental monitoring system is increasing, but because of the technology of China's environmental monitoring is relatively backward, later Maintenance work need to lots of manpower and material [1].

With the development of GSM (Global System for Mobile Communications), GPRS (General Packet Radio Service) technology is getting mature [2], it provides a way for wireless Internet access. Through the existing GPRS communication network, the equipment can exchange data with data center on the Internet by the Internet service, so as to facilitate the simultaneous monitoring of scattered geographical monitoring, in addition to, the installation of equipment is very easy, and the cost of installation is low.

In this paper, an environment monitoring system based on GPRS and ARM is designed to real-time monitor the temperature, humidity and the concentration of SO2 of remote air by wireless.

\section{System Structure}

The system includes three parts: the monitor, GPRS communication network and the software of monitoring center. The monitor is distributed in the area to be monitored. It is responsible for collecting the ambient temperature, humidity and the concentration of SO2,and sends those information to the software of monitoring center by using GPRS and Internet network as the communication carrier, if the value of environment exceeds the threshold, the monitor will sent alarm SMS to the mobile phones of monitoring staff. The software of monitoring center can display real-time data, query historical data, analysis and print chart, and so on. The structure of system is shown in Figure 1: 


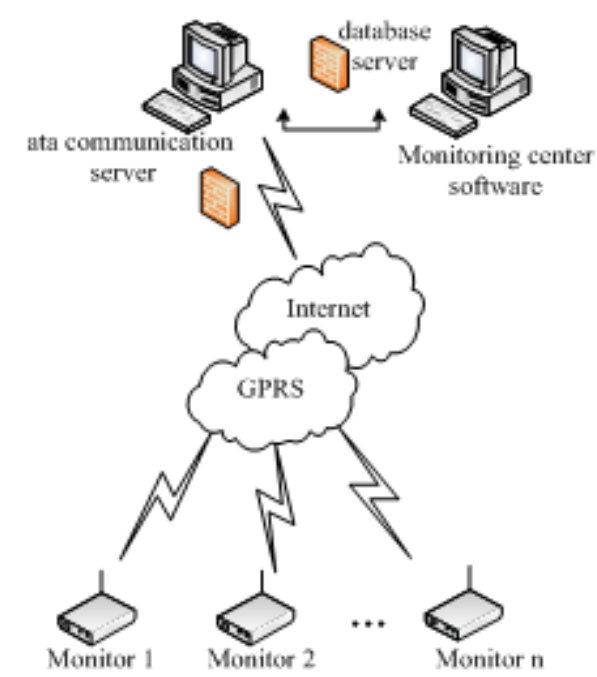

Figure 1 System structure

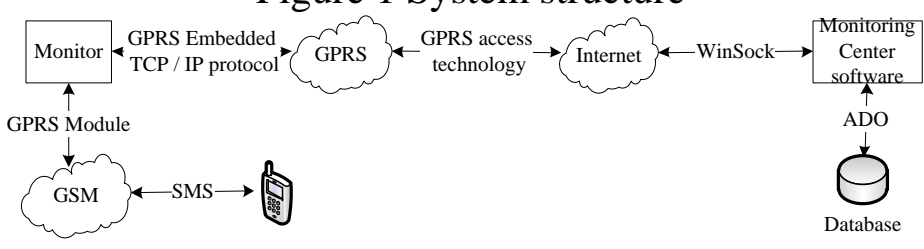

Figure 2 Transmission of data between networks

It is the key part of the environment monitoring system to realize real-time communication in different networks. Data transmission between the networks is shown in Figure 2, firstly the sensor in monitoring instrument collects environment data, then GPRS module will send data to the GPRS network, secondly GPRS network connect to the Internet network through GPRS access technology, data will be sent from Internet network to the software of monitoring center through WinSock technology, the software of monitoring center access the database by using ADO, and the final data is stored in the database. At the same time if the environmental data exceeds the threshold, GPRS module will send alarm SMS to the phone of the designated monitoring staff through the GSM network.

\section{The design of system hardware}

The hardware design of the system is the hardware design of the monitor. The hardware design of the monitor mainly depends on the rich peripheral interface of LPC2220.the PCB board of Monitor is a self-made, double-layer and industrial-grade circuit board, the structure of monitor is shown in Figure 3, which is divided into three parts according to the functions, they are the main control part, the signal acquisition part and communication part.

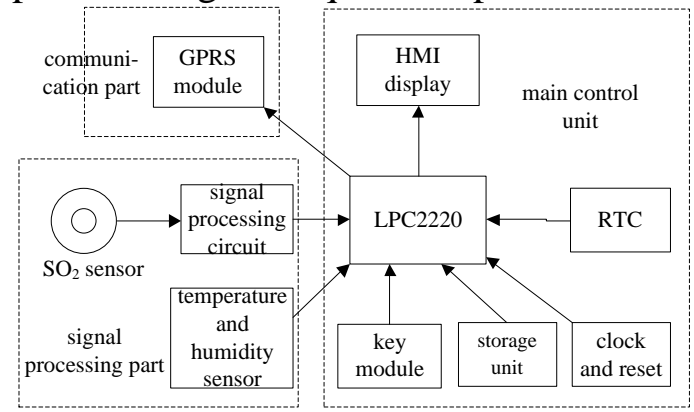

Figure 3 the structure of monitor

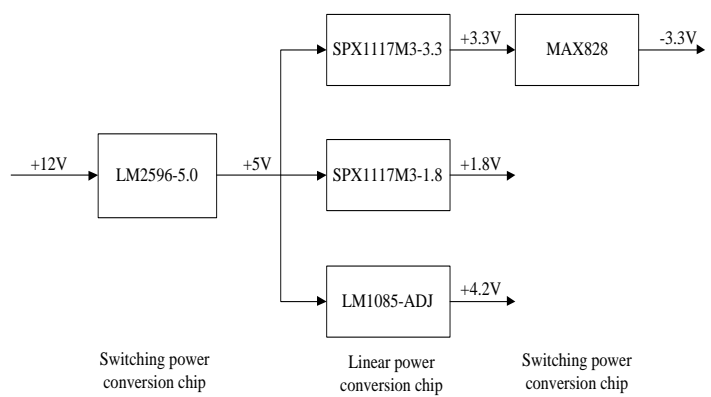

Figure 4 the structure of power transform module

Several key components of the monitor are as follows:

Microprocessor: The microprocessor uses LPC2220 produced by NXP, it is rich in peripheral interfaces through providing up to 76 GPIO configuration, besides LPC2220 can encrypt the codes, 
which is high security because of that the code is difficult to be cured after reverse deciphering.

Storage Unit: In order to meet the requirements of memory capacity and processing speed, the processor needs to expand the memory. According to the functional requirements and commonly used minimum system of LPC2220, the processor can expand 512KB of SRAM and 1MB of Flash. SRAM uses IS61LV25616AL, Flash uses SST39VF1601, which is very fast.

GPRS module: SIM300CZ which is produced by SIMCOM is used for GPRS module. The module is embedded with powerful TCP / IP protocol stack, and can highly-speed transmit SMS and data with low power consumption. LPC2220 sends AT commands to the GPRS module through serial port UART1.

Sensor: the sensor which is used collecting the concentration of SO2 uses SO2 / CF-20 type electrochemical gas sensor produced by MEMBRAPOR, the accuracy of which can reach 0.2PPM.The temperature and humidity sensors use the digital sensor integrated temperature and humidity produced by SENSIRION, the sensor accuracy of up to 12bit using for temperature and 14bit using for humidity, the range of temperature measurement is from $-40{ }^{\circ} \mathrm{C}$ to $123.8{ }^{\circ} \mathrm{C}$.

Power transform module: In order to ensure low interference, low heat dissipation and efficient power utilization, the system uses a power supply which integrates linear power supply and switching power supply, the structure of power transform module is shown in Figure 4.

\section{The design of system software}

The design of system software includes and the software design of the monitoring center, the overall process of the software design of the monitor is shown in Figure 5.

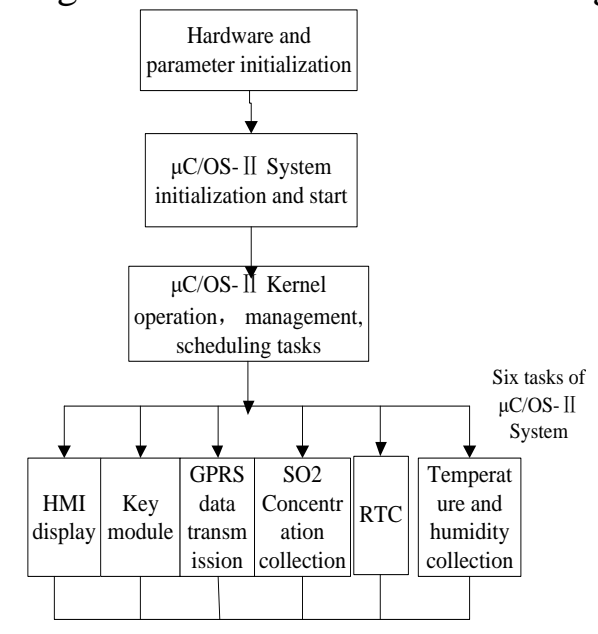

Figure 5 the overall process of the software design of the monitor

\begin{tabular}{|c|c|c|c|c|c|c|}
\hline Start & ID & Time & Tem & Hum & $\mathrm{SO}_{2}$ & End \\
\hline 1Byte & 3Byte & 12Byte & 5Byte & 4Byte & 3Byte & 1Byte \\
\hline
\end{tabular}

Figure 6 the format of packet

\section{The design of real - time task}

The system creates 6 tasks, they are HMI display task Task0Stk_DIS, and key task Task1Stk_KEY, and GPRS transmission task Task2Stk_GPRS, collection task of concentration of SO2 Task3Stk_SO2, collection task of temperature and humidity Task4Stk_SHT11, RTC task Task5Stk_SMS. The data length of each task is not the same, the data stack of display task and GPRS transmission task are larger, allocated 512 bytes, the length of other tasks is 128 bytes. The main program code is:

$$
\text { int main (void) }
$$

OSInit (); 


\author{
SYSTEM_Ini (); \\ OSTaskCreate (Task0Stk_DIS,(void*)0,\&TaskStk[TaskStkSize -1],7); \\ OSTaskCreate (Task1Stk_KEY,(void*)0,\&TaskStk1[TaskStkSize -1],6); \\ OSTaskCreate (Task2Stk_GPRS,(void*)0,\&TaskStk[TaskStkSize -1],5) \\ OSTaskCreate (Task3Stk_SO2,(void*)0,\&TaskStk[TaskStkSize -1],4); \\ OSTaskCreate (Task4Stk_SHT11,(void*)0,\&TaskStk[TaskStkSize -1],3); \\ OSTaskCreate (Task5Stk_RTC,(void*)0,\&TaskStk[TaskStkSize -1],2); \\ OSStart(); \\ return 0 ; \\ \}
}

\title{
The design of communication protocol
}

LPC2220 communicates with the GPRS module through the serial port UART1, the baud rate of which is 115200, the data frame for which is 8 data bits and a stop bit, in order to achieve real-time data transmission, the protocol uses UDP as a transport layer protocol, the format of application layer packets is shown in Figure 6.

The Start character of packet is "*", the corresponding hexadecimal of which is $2 \mathrm{AH}$. The End character is "\#", the corresponding hexadecimal which is $23 \mathrm{H}$. ID refers to the ID number of the monitor, the size of which is 3Byte, its format is III, such as: 001, meaning No. 1 monitor. Time refers to the start time of data acquisition, the size of which is 12Byte, its format YYMMDDHHMMSS, such as: 091012121530 that means 12:15:30 October 2009. Tem refers to the temperature of ambient ,the size of which is 5Byte, its format is FTTTT, F represents the sign bit, such as: 12021 means negative $20.21{ }^{\circ} \mathrm{C}$. Hum refers to the humidity of ambient, the size of which is 4Byte, and its format is $\mathrm{HHHH}$, such as: 5012 means $50.12 \%$. SO2 refers to the concentration of SO2, the size of which is 3Byte; its format is SSS, such as: 104 mean 10.4PPM.

\section{Software Filtering Algorithm of Environmental Data Processing}

The sensor will be affected by external electromagnetic interference and circuit interference, in addition to anti-jamming measures of hardware; software filtering can further improve the accuracy of collecting data. in order to improve the accuracy of the data, the sensor collects data every 3 seconds, and continuous acquisites 10 data which are C1 C10, then gets the actual data S by computering the median of this group data, the monitor data processing cycle is 30 seconds.

If the monitor is set to send data every $M$ minutes, the monitor will work $Z=M * 60 / 30$ cycles, and get $\mathrm{Z}$ data which are S1 SZ, take arithmetic median filtering method on Z data to get, lastly send data $\mathrm{H}$.

\section{The design of Monitoring Center Software}

Monitoring Center software runs on the monitoring center server of remote Internet, the operating environment for which is Windows XP, completes of recepting and processing data about air quality. Software bases on VC + + and MYSQL, it is a server in the communication process with long-range environmental monitoring device, and uses WinSock to receive data, the system using MySQL database provides more powerful processing and management of data, which made view, analysis and print real-time Monitoring data convenient.

\section{Conclusion}

In this paper, an environment monitoring system based on GPRS and ARM is designed, which integrates the related technologies of embedded technology, sensing technology, wireless 
communication technology and computer application technology. Although the system only monitors concentration of SO2, temperature and humidity, but the system interface is rich, accessing to other sensors can achieve the environmental monitor, such as CO2, particulate matter. The environmental monitoring system links all isolated environmental monitoring points into a large environmental monitoring network through existing GPRS and Internet networks, It has the characteristics of wireless automation, high real-time performance, easy installation and low maintenance cost, provides a more effective way of monitoring.

\section{Acknowledgements}

Project name: Research on intelligent logistics distribution scheme based on Internet of things Project ID: 2014Q020

\section{References}

[1]Wu Feng, Li Cheng tie and so on. Research on remote monitoring system based on Web technology [J]. Instrumentation reported, 2005, 8: 241-243.

[2] Chang Xiong, Zhou Xu. Design of Intelligent Terminal Based on GPRS in Information Collection System [J]. Computer Technology and Development, 2005, 8: 154-158.

[3] Zhou Li gong. ARM embedded system foundation tutorial [M]. Beijing: Beijing University of Aeronautics and Astronautics Press, 2005.

[4]Design of embedded remote data acquisition system based on GPRS [J]. Computer Technology and Application, 2009, (1): 127-129. 